Argonne

ANL/DIS-21/6

\title{
Oregon Rivers Characterization Tool
}

Technical Report

Decision and Infrastructure Sciences Division 


\title{
Acknowledgement
}

This report has been prepared by Argonne National Laboratory (Argonne). Argonne is a U.S. Department of Energy laboratory managed by UChicago Argonne, LLC under contract DE-AC02-06CH11357. This study was sponsored by the Cybersecurity and Infrastructure Security Agency (CISA) Regional Resiliency Assessment Program. We would like to thank Patrick Massey, Chass Jones, Jason Osleson and Jaysen Goodwin at CISA for their support and guidance throughout this effort.

\author{
About Argonne National Laboratory \\ Argonne is a U.S. Department of Energy laboratory managed by UChicago Argonne, LLC \\ under contract DE-AC02-06CH11357. The Laboratory's main facility is outside Chicago, at \\ 9700 South Cass Avenue, Argonne, Illinois 60439. For information about Argonne \\ and its pioneering science and technology programs, see www.anl.gov.
}

\section{DOCUMENT AVAILABILITY}

Online Access: U.S. Department of Energy (DOE) reports produced after 1991 and a growing number of pre-1991 documents are available free at OSTI.GOV (http://www.osti.gov/), a service of the US Dept. of Energy's Office of Scientific and Technical Information.

Reports not in digital format may be purchased by the public from the National Technical Information Service (NTIS):

U.S. Department of Commerce

National Technical Information Service

5301 Shawnee Rd

Alexandria, VA 22312

www.ntis.gov

Phone: (800) 553-NTIS (6847) or (703) 605-6000

Fax: (703) 605-6900

Email: orders@ntis.gov

Reports not in digital format are available to DOE and DOE contractors from the

Office of Scientific and Technical Information (OSTI):

U.S. Department of Energy

Office of Scientific and Technical Information

P.O. Box 62

Oak Ridge, TN 37831-0062

www.osti.gov

Phone: (865) 576-8401

Fax: (865) 576-5728

Email: reports@osti.gov 


\section{Oregon Rivers Characterization Tool}

Technical Report

Eugene Yan and Jeremy Feinstein

Environmental Sciences Division, Argonne National Laboratory

Thomas Wall

Decision and Infrastructure Sciences Division, Argonne National Laboratory

August 2021 


\section{Oregon Rivers Characterization Tool}

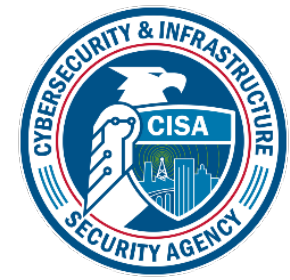

August 2021 


\section{Table of Contents}

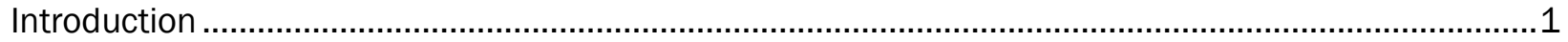

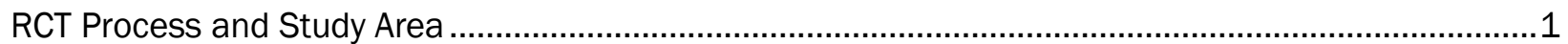

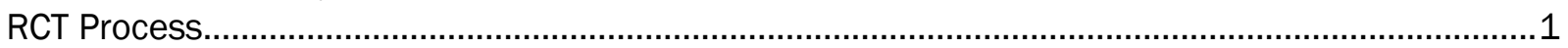

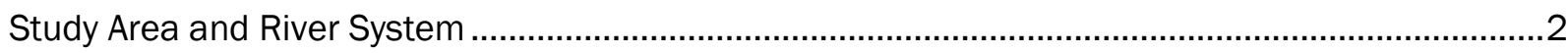

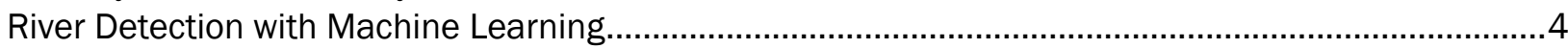

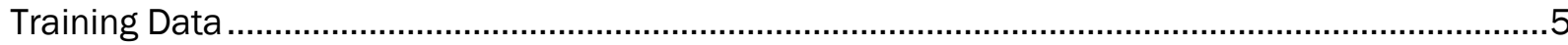

Inputs

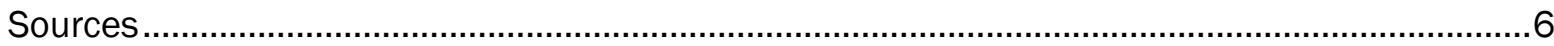

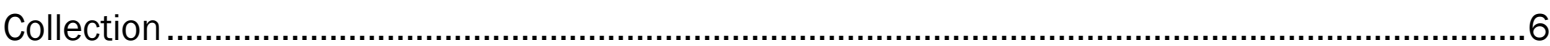

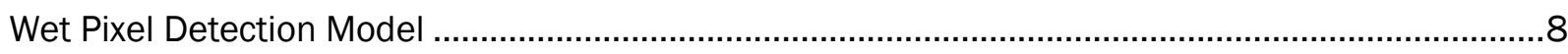

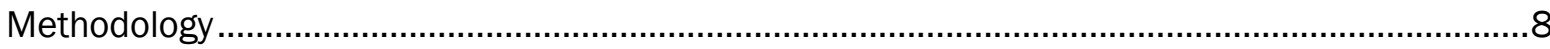

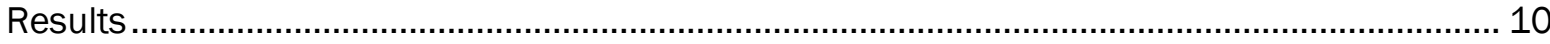

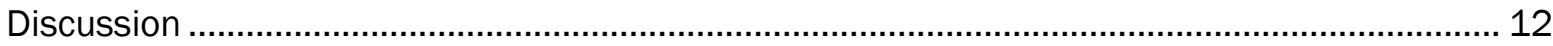

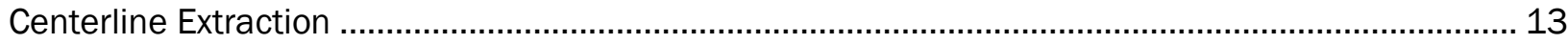

Channel Width Characterization .............................................................................................. 14

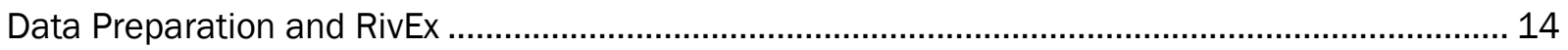

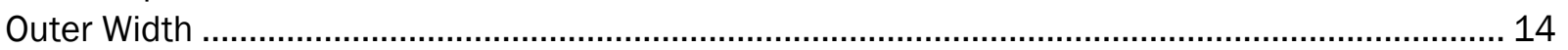

Near-branch Width Filtering .................................................................................................. 15

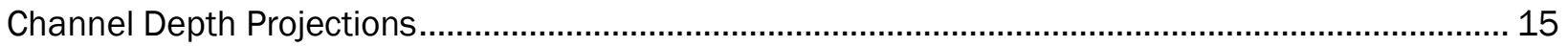

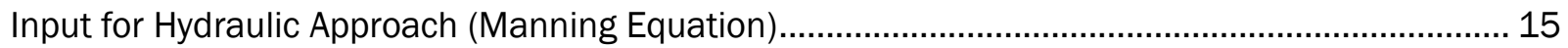

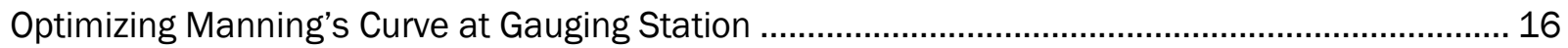

Predicting Measuring Point Depths ..................................................................................... 18

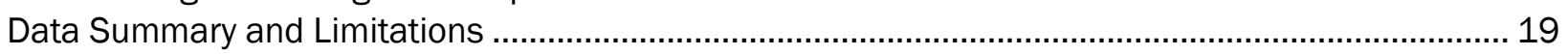

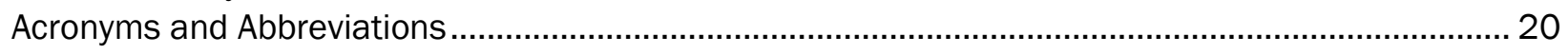

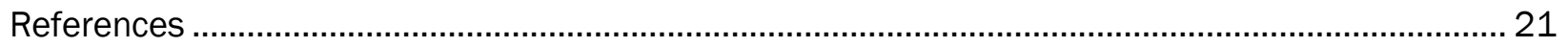




\section{Introduction}

DHS's Regional Resiliency Assessment Program (RRAP) undertook the Oregon State Transportation Systems RRAP project between 2018-2021 to assess the seismic vulnerabilities of the state's transportation system to a Cascadia Subduction Zone (CSZ) earthquake (CISA 2021). The project primarily focused on the effects of a CSZ earthquake on the mobility of emergency response efforts into the region. In the event that bridges located at crucial river crossings fail during a CSZ earthquake, rivers will become natural barriers affecting the ability to support response and recovery efforts. The characteristics of rivers (e.g., width, depth, and flow) are critical data to inform planning for the recovery of transportation systems across river barriers and determine reopening times following a CSZ earthquake. As part of the transportation system assessment, an Argonne National Laboratory (Argonne) project team developed a River Characterization Tool (RCT) to quantify the physical characteristics of the river system in Oregon, which can be used to inform a system-level analysis of transportation recovery and reopening times. This report mainly discusses approaches, methods, development, and applications of the RCT. The use of the river data generated from this tool for a system-level analysis of transportation recovery is discussed in the Oregon Transportation Systems RRAP Resiliency Assessment report (CISA 2021).

The primary goal of the RCT is to generate statewide dataset of widths, depths, and flows for all rivers across Oregon to assess the effects of river barriers on emergency response and recovery efforts following a CSZ earthquake. The RCT leverages remote sensing data that multispectral sensors collect from satellites, employs a deep learning model to characterize river predictions, and computes river channel geometric dimensions with hydraulic relationships and river predictions. The entire process of river characterization is automated and implemented through the RCT.

\section{RCT Process and Study Area}

The characterization of river dimensions has been traditionally performed through in-situ measurements, which are labor intensive, time-consuming, and cost prohibitive for large-scale implementation. This project aims to develop a RCT with a more robust process that builds on existing water body extraction methods, improving results with a deep learning approach. This section presents the RCT process and approach as well as the study area used for application of the RCT.

\section{RCT Process}

A RCT process is designed to characterize river geometric dimensions through four sequential data analysis and modeling components: (1) river detection with machine learning approach, (2) river centerline extraction, (3) channel width calculation, and (4) channel depth optimization (figure 1). All components in this process are automated and implemented in the RCT.

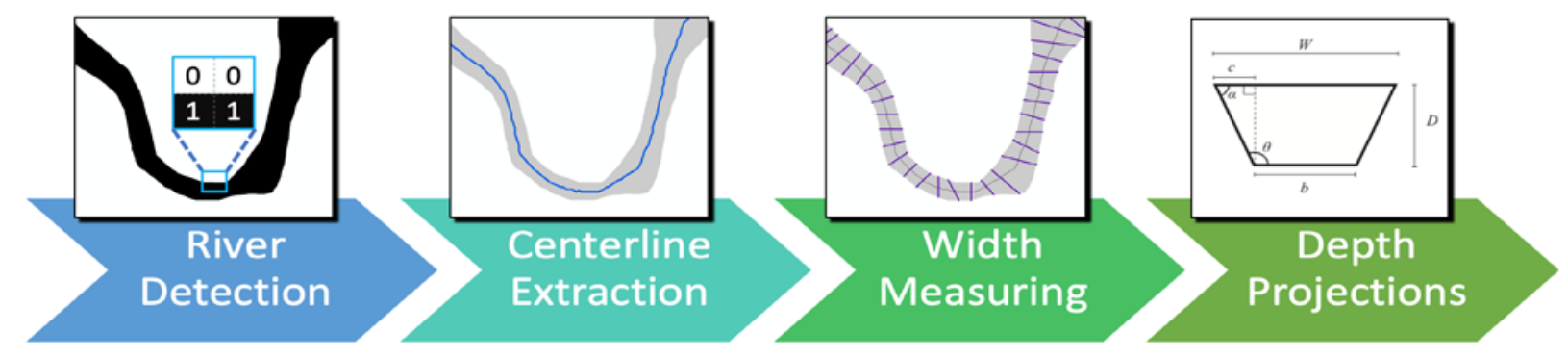

FIGURE 1.-River Channel Characterization. 
Detection of river water bodies is achieved using a remote sensing approach. However, identifying the presence of water is affected by many factors (e.g. terrain effects and features with similar spectral characteristics). In this project, the research team developed a supervised machine learning model to produce a binary river mask indicating water presence or absence. The river detection model, which is a composite of three separate artificial intelligence algorithms, was trained on multispectral sensor data from various sample areas including water and non-water pixels. The model improves the prediction of water presence (via wet pixel detection) by incorporating its nuanced understanding of which spatial and spectrometric patterns correlate with wetted pixels that form a stream network.

River centerline extraction is a prerequisite for characterization of channel width. Obtaining a centerline is done through Python image processing libraries, and then vectorized and filtered with graph and network analysis tools.

Channel widths are calculated using the length of segments drawn orthogonally to centerlines up to channel boundaries (i.e., non-wetted pixels) at evenly spaced points along river reaches. The resulting estimated channel widths then inform trapezoidal generalizations of river cross-sections which, along with measurement data collected from U.S. Geological Survey (USGS) gauges and regional extrapolation, are used to optimize depth approximations.

River channel characterization is performed through four sequential analysis and modeling components. First, trained machine learning models are used to produce a binary water presence map with satellite data; second, a water presence map is then thinned, vectorized, and filtered with network analysis to extract river centerline; third, river width is estimated for line segments that are drawn perpendicularly to a centerline; and lastly, channel depths are projected by a Manning-based model with data from USGS gaging stations.

\section{Study Area and River System}

The RCT developed in this project is applied as a regional assessment for all rivers in Oregon. In determining the appropriate approach for collecting training data, constructing machine learning models, and employing regional geometric relationships, understanding characteristics and variation of topographic, physical, and geologic setting and features across the region is important. Castro (1997) identified the significant role of physiography affecting bedload, channel bank, and channel bed characteristics when evaluating regional relationships between river geometry in the Pacific Northwest. Fenneman and Johnson (1946) have developed a multi-tier physiographic classification system for classifying regions with similar rock types, topographies and geomorphological histories. As a hierarchy classification from the general to the specific, they categorize spatial extents across the United States into physiographic divisions, provinces, and sections. Bieger et al. (2015) further used Fenneman's province-level classification system for creating regional coefficients for river geometry relationships across the United States. In this study, the research team also employed Fenneman's physiographic provinces both for comprehending geologic diversity across the study area, and guiding training data collection. 


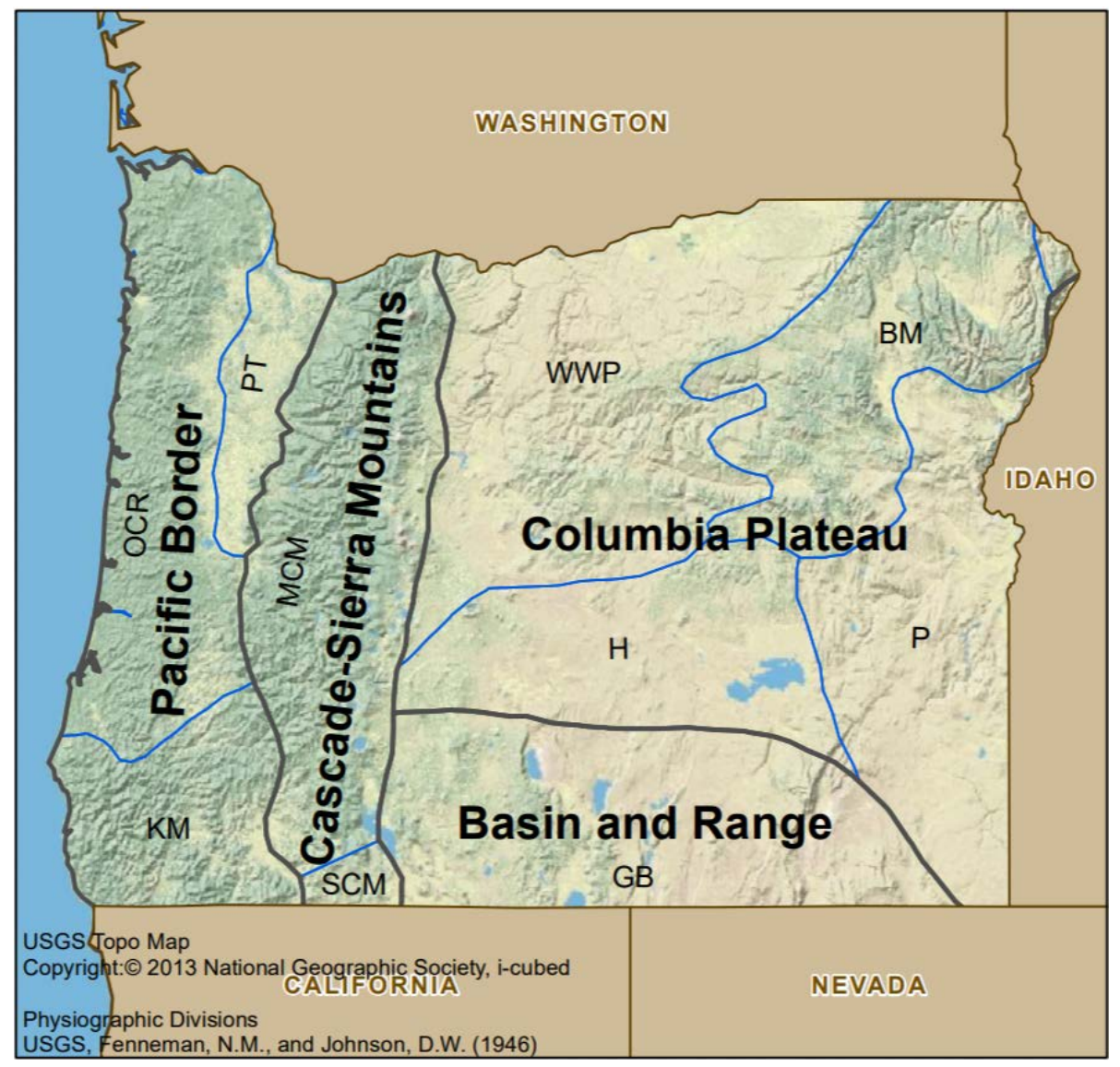

FIGURE 2.-Oregon's Physiographic Provinces. ${ }^{1}$

Oregon lies within the Intermontane Plateau, Pacific Mountain System, and Rocky Mountain System divisions, though the latter constitutes only the Northern Rocky Mountains province in an insignificant proportion (figure 2).

The Intermontane Plateau division of Oregon consists of the Basin and Range province and the Columbia Plateau province. The Columbia Plateau is distinguished by its substratum formed from massive basaltic lava flows (Fenneman 1928). Across the plateau are networks of small streams, the eastern half of which feed into the Snake River along the northern part of Oregon's eastern boundary. As the Snake River makes its way into southern Washington, it outlets into the Columbia River, which descends until the Oregon-Washington border and then heads westward to the Pacific Ocean. The rest of the plateau can generally be described as small rivers which feed major veins across the high plains, like the Umatilla, John Day, and Deschutes Rivers, which head north and merge into the Columbia River. Some water systems, like the Harney section, have no stable outflows and constitute a closed watershed that feeds Harney Lake.

\footnotetext{
${ }^{1}$ Oregon's physiographic provinces as defined by Fenneman, N.M., and Johnson, D.W. Grey lines represent province boundaries; blue lines represent section boundaries. Sections are abbreviated (OCR = Oregon Coastal Range; KM = Klamath Mountains; PT = Puget Trough; MCM = Middle Cascade Mountains; SCM = Southern Cascade Mountains; WWP = Walla Wall Plateau; $\mathrm{H}=$ Harney; BM = Blue Mountain; $\mathrm{P}=$ Payette; GB = Great Basin)
} 
The Basin and Range province is characteristically made up of isolated, uniform, and parallel mountain ranges, the result of long periods of extensional deformation in the Great Basin (Fenneman 1928). The northwestern edge of the Basin and Range province only has these parallel features in subtlety, but the inter-province boundary line is informed by the Plateau's substratum of lava and the Basin's isolated mountain-valley formations. The unique and isolated features of the Basin and Range province create many closed watersheds, forming endorheic bodies with no major outflow to the Pacific such as Lake Summer, Lake Albert, and Lake Hart.

The Pacific Mountain System includes the Pacific Border and the Cascade-Sierra Mountain provinces. The inland Cascade-Sierra constitute mainly north-south alpine mountains and stratovolcano formations. In Oregon, most of the Cascade-Sierra is occupied by the Middle Cascade Mountain section. Initially formed through crustal uplift, the Middle Cascade's high altitudes have developed from extensive accumulation of volcanic cover over time. Most streams in the CascadeSierra run perpendicular to the mountain range, with a distinct east-west pattern. The Cascade's watershed is divided medially by some of Oregon's tallest peaks. The eastern division generally brings water to the Deschutes River in the Columbia Plateau, or to the Klamath, which heads south out of the State. The western part carries much of its discharge into the Pacific Border's Willamette Valley, whose river heads north to outlet into the Columbia. Rivers in the western watershed which do not descend into the valley instead head straight for the Pacific, discharging into the Umpqua and Rogue Rivers on the way.

Situated on the western rim of the country, the Pacific Border province pulls in major precipitation from winter storm over the Pacific Ocean (Castro 1997). The northern constituent of the Pacific Border province is the Oregon Coastal Range. Hardly mountainous, the coastal range section is described by Fenneman as "range-like." South of the Oregon Coastal Range section is the Klamath Mountains section, distinguished by old, robust rock. The streams in the Puget Trough section take the Willamette River up to the Columbia. The rest of the many small streams across the Pacific Border generally head directly for the Pacific, either merging into some of the province's larger substituents (the Rogue and Umpqua rivers), the medium-sized ones (Coquille, Alsea, Siletz, Siuslaw), or heading there directly.

\section{River Detection with Machine Learning}

A primary goal of the river detection tool is to create a binary prediction map of rivers across the diverse study area described in the previous section. This section will provide a brief overview of the current, widely used methods for river detection, machine learning methods that can be leveraged to overcome the existing challenges in river detection, and an in-depth discussion of the wet pixel detection model developed for this project. Figure 3 shows a procedure for the river detection model was developed and tested.

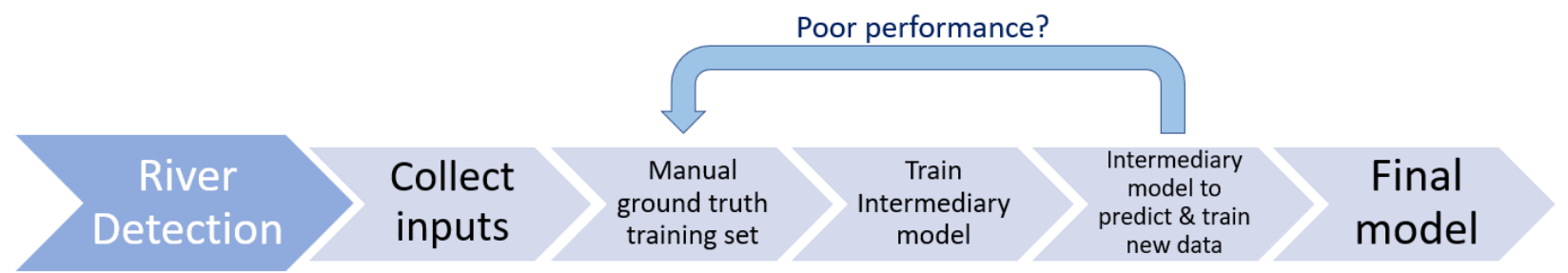

FIGURE 3.-Flow Diagram for River Detection Procedure. 
The classification of surface features is a long-standing goal in remote sensing. Water body extraction has often relied on the use of normalized difference water index (NDWI), proposed by McFeeters (1996), and the improved, modified NDWI (mNDWI), demonstrated a decade later by Xu (2006). These calculations were built on the strong absorption of infrared light by water to distinguish lakes, rivers, and floodwater from terrain with high infrared reflectance, like vegetation (McFeeters 1996). While a threshold approach for creating land-water masks from NDWI can identify water bodies having values greater than zero, turbidity, vegetation health, and various biogeochemical properties of a terrain often make threshold selection variable and inconsistent across time and space (Huang 2018). More troubling, NDWI and mNDWI are prone to misclassify mountain shadows, various pavements, building rooftops, snow cover, and other terrain (Acharya et al 2018). Uncertainties in spectral-based approaches thus fuel concerns when applied across a large, diverse study area where river channels have frequent passage through mountainous terrain or urban areas.

In this project, the research team aimed to overcome typical problems associated with spectralbased water indices by employing a machine learning approach. Deep neural networks can enhance classification outcome by recognizing spatial patterns and reinforcing learned, featured connectedness. For this project, three neural network were combined to employ a hybridized neural network.

\section{Training Data}

One of the most critical prerequisites in developing a successful model is establishing a training dataset that represents water presence under a wide range of terrain effects and interfering features. This section provides an overview of the methods to develop training datasets, data source, and collection methods.

\section{Inputs}

Visible light spectrum images (red, green, blue) and infrared spectrum images (near infrared and 2 short-wave infrared bands). Extensive testing found that slope information could supplement NDWI data to improve recognition of water bodies and reduce some terrain effects. The elevation model suggested that many river reaches had locally consistent elevations and neighboring banks that sat higher than the river and had increased slope values. To parameterize this observation, Equation 1 was used to create a combined NDWI-slope input.

$$
\begin{aligned}
& \text { Combined }=N D W I-1.5 * \text { slope } \\
& \text { where clip }(\text { Combined },[0,1]) \rightarrow \max (0, \min (\text { Combined }, 1))
\end{aligned}
$$

Further testing of preliminary exploratory models also identified that some errors arise from misclassifying roadways as river water. Roadways sometimes have similar spatial and spectral patterns as rivers and occasionally run parallel along river reaches. In order to minimize the misclassification, two additional layers were added into input datasets: rasterized road and USGS national hydrography dataset (NHD) flowline data representing river reaches. Though the flowline input layer's accuracy does not match the level the research team hoped to achieve in constructing the training input dataset, the multiple layers of datasets in conjunction helped decrease errors from misclassification.

In a summary, data involved in the preparation and deployment of a machine learning model is thus categorized as: (1) multispectral photometry, in the form of an NDWI, as a main basis for pixel 
prediction; (2) elevation and slope to filter and guide classification, and (3) surface feature (vector) information to increase accuracy across terrain with similar reflectance levels.

\section{Sources}

The research team collected spectral data for the model from public satellite data. The Sentinel-2 mission provides the highest resolution, publicly accessible method for obtaining surface reflection intensities in the red, green, blue, near infrared (NIR), and two shortwave infrared (SWIR) spectral ranges. The mission's low revisit duration of five days enables researchers to compute temporal raster cell averages from multiple observations in a short measurement window. For the initial collection of training data, ClimateEngine was used to target and download spectral data at specific sites across the area of interest (AOI). For the heavier task of full-AOI data collection, Google Earth Engine allows researchers to filter imagery by percent cloud coverage (using a third SWIR spectral range) and to download data directly to Google Cloud.

Elevation data for the AOI was downloaded from the U.S. Department of Agriculture's Geospatial Data Gateway and ArcMap's "Slope" toolbox was used to generate slopes, which were subsequently resampled to match the cell size and position of the Sentinel-2 raster grid. Features for flowline geometry, from the NHDPlus Version 2, and road geometry were rasterized on the Sentinel-2 raster grid.

\section{Collection}

Data was collected from these sources in multiple phases as part of the active learning process (explained later in the Methodology section). The initial phase of data collection focused mainly on gathering inputs in each major physiographic region to capture a diverse range of spectral reflectance patterns across Oregon's diverse topography. Input samples were selected to target each of the features including river features. The research team also collected supplementary inputs which focused mainly on regions prone to mountain shadow, since mountain shadows create common problems in water classification. 


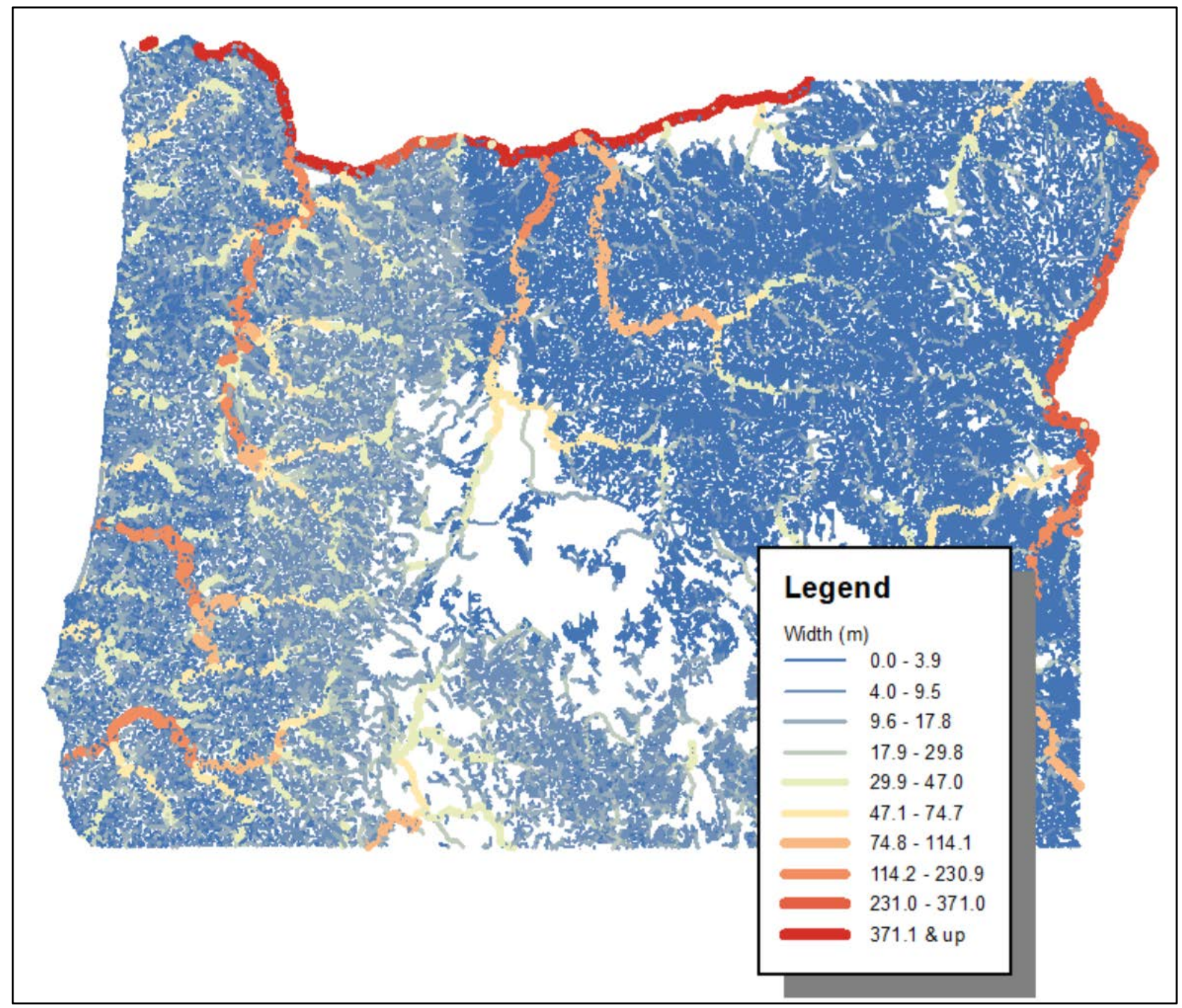

FIGURE 4.-NHD Flowlines Plotted according to Their Projected Channel Widths Using Physiographic Province-level Regression Coefficients Derived by Bieger et al (2015).

In the second phase of data collection, more advanced techniques were used to ensure that training data contained a variety of rivers with a diverse range of channel widths. Bieger et al. (2015) aggregated extensive datasets of river geometries across the United States, which could be used to calculate hydraulic geometry power law relationships in each physiographic province. The research team used Bieger et al.'s derived regression coefficients to construct a map of river widths across Oregon (figure 4), and used this map to guide a collection of samples containing a variety of representative river widths on both east and west sides of the State.

This two-phase approach guaranteed robust training data with imagery from each physiographic region, shadowed river reaches, and a variety of river widths across the state. 
Wet Pixel Detection Model

Methodology

The Wet Pixel Detection Model (WPDM) is a deep neural network pipeline that consists of a deep encoder-decoder model and a deep convolutional neural network classifier for pixel-level and patchlevel binary classification tasks, respectively. The research team employed the state-of-the-art encoder-decoder model, where the high-level semantic features containing information of the shape and connectedness of the river are extracted by the encoder and fed directly into the decoder, and the low-level fine-grained features are passed to the decoder through a skip connection, to enhance the overall quality of the pixel-level probabilistic prediction. The output of the encoder-decoder model is sensitive to the inputs' variations due to the skip connection design, and therefore prone to noise. To address this problem, the research team further employed a binary classifier that labeled input patches as water-present (positive) or water-absent (negative) and was trained with a cost-sensitive loss in favor of positive prediction. The quality of the output was improved by classical postprocessing techniques.

The research team conducted a thorough performance comparison across the state-of-the-art architecture designs for the deep encoder-decoder model for this task: U-Net, DU-Net, UNet++, R2UNet, AttUNet, NestedUNet, Unet++, EDSR, and Mask-RCNN. The experimental results showed that UNet++ performed competitively against all other designs. For the classifier, the research team took a vanilla discriminator of SrGAN with the first and last layer modified to adapt the shapes of inputs and labels.

The satellite map of the AOI is a high-resolution image with millions of pixels. To generate output at the same high resolution for assessing river width, a deep learning model would require high computational time. Current state of the art models (Brock et al. 2019) produce high-fidelity outputs up to a resolution of 512 x 512 pixels, which takes Google's TPU v3 Pod up to 2 days to train. In this study, to decrease training time, the input map is divided into smaller patches and wet pixels are predicted at the patch level. Intuitively, there is a trade-off between localization accuracy and the context of a patch. Larger patches require additional max-pooling layers, which in turn reduce localization accuracy, while small patches limit the spatial context available to the network. The WPDM uses patches of $64 \times 64$; modern deep neural network (DNN) structures work well for such images on representation learning (Radford et al., 2016).

The training of the network was performed on pre-processed input patches and their corresponding ground truths. Random sampling of the training data would result in pessimistic predictions from the detector, as most of the model domain is land (i.e., non-water) and encoded in the ground truth as zero. On the other hand, sampling too many patches that contain river reach pixels may create an optimistic model. Spurious factors of variations on some pixels in non-river input patches, which accidentally correlate with the prediction, can mislead the model to make optimistic predictions on all the neighborhood pixels.

The final model used a proposed pyramid prediction scheme. The process of river detection was divided into a low-level classification task (the linear classifier) and a high-level prediction task (the feature extractor and enhancer). Each patch was first pushed through the binary classifier to determine if the patch contains any water. If positive, the patch was further pushed into the detector to refine the prediction on a pixel level. To balance training data and reduce pessimistic predictions, half of the training patches were randomly sampled from the ground truth data while the other half were sampled such that at least 3 percent of the patch was made of water pixels. Eighty percent of the training data developed through a manual ground truth classification and labeling (or ground 
truthing) process was used in the training of the model, while 20 percent was used to generate performance metrics when the final model was complete.

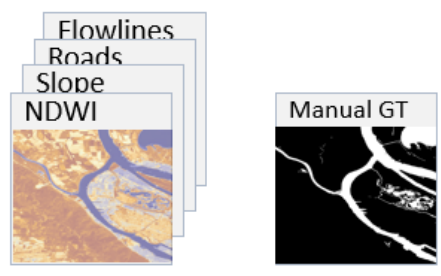

Training data

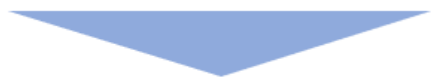

\$4 "Patching"

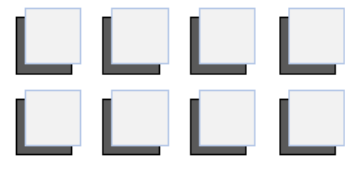

$64 \times 64$ samples

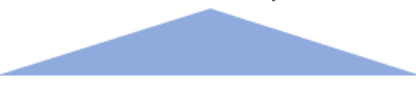

New GT

Correct model output to compile additional training data
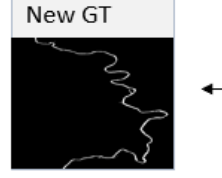

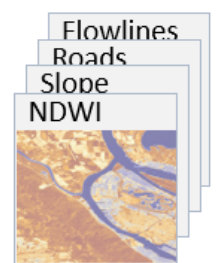

Novel untested data

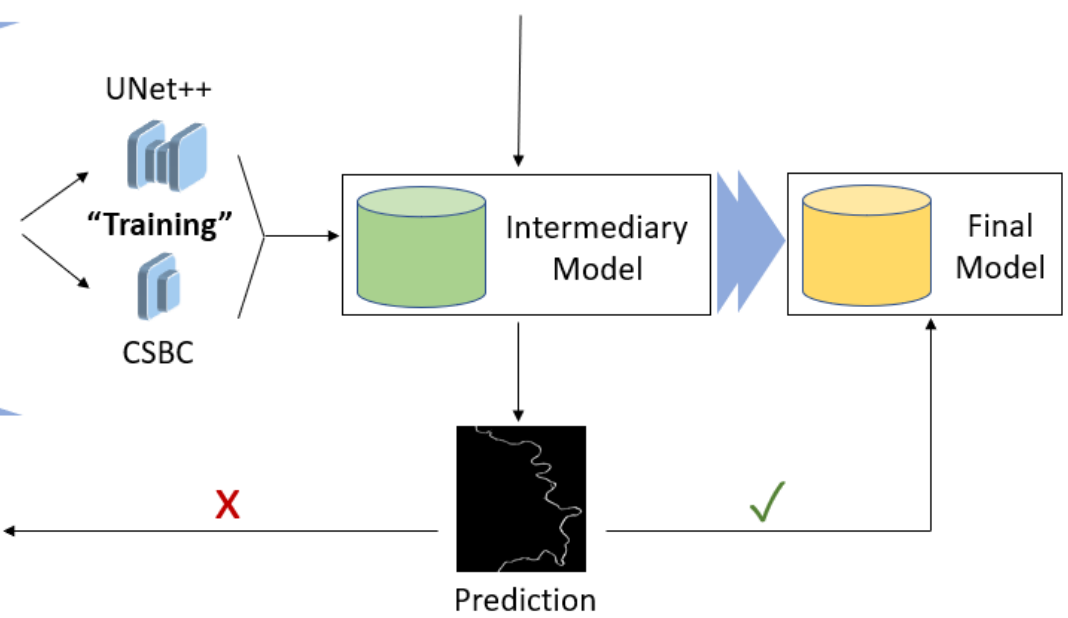

(Examined manually for quality)

FIGURE 5.-Overview of Training Process.

Figure 5 shows an overview of the training process for the machine learning model, which ultimately predicts river pixels within the entire study area. A training data set was prepared, patched, and used to train UNet++ and cost sensitive binary classifier neural networks. An intermediary model was then generated and evaluated against new, untested training data. If the prediction results were unsatisfactory (as determined by human), the results were corrected, patched, and then added to the existing training set, constituting an "active learning" training process.

The development of ground truth data is divided into two phases. Both phases of ground truth labeling use Google Earth Pro reference imagery, along with the satellite inputs themselves, to manually classify pixels as water (1) or non-water (0). Manual ground truth labeling is completed using the free image editing software GIMP. Dividing data collection into two phases allows a draft model (trained on data from the first phase) to predict the ground truth values for the following set of training data. Though an intermediary model could predict outputs that deviate from ground truth, efforts can be optimally tailored to areas with greater differences, reducing time invested in developing superfluous ground truth. This technique, a variant known as "active learning," allows an intermediary deep learning model to assist in the creation of additional training data. This process can be repeated to expand the training data and improve intermediary models until the final model is selected.

The initial collection of training data was done across Oregon's four dominant physiographic regions. After the draft model was deployed, additional training data were gathered, guided by a map of 
approximate river widths calculated using hydraulic geometry power law relationships derived by Bieger et al. (2015) to target smaller streams, whose topographies are more diverse than main river reaches.

Results

The WPDM was used to infer pixels over input imagery occupying a $47947 \times 91732$ pixel map. The research team demonstrated the proof-of-concept machine learning model results against standard spectral-index methods. They then provided standard statistical metrics generated by comparing predictions performed on the testing set (the 20 percent of input data excluded from the model training process) to their manual ground truth. Finally, the team demonstrated improvements in the model's predicting capabilities made by the active learning process through showing model predictions in the same region before and after the active learning process.

Proof-of-Concept

A primary goal of a deep learning application for the wet pixel detection model is to improve the water-sensing prediction capabilities widely available, such as NDWI and mNDWI. This section reviews a sample extent within Oregon and compares results from a binarized NDWI raster map against the WPDM output. This comparison demonstrates the applicability of deep learning in the problem domain. Figure 6 presents these results. For a comparable NDWI result, the research team adjusted the tolerance threshold to account for the pessimistic result of water presence when using the standard threshold of zero. The chosen threshold for this binary map was -0.2, even though certain river pixels still have negative results. 


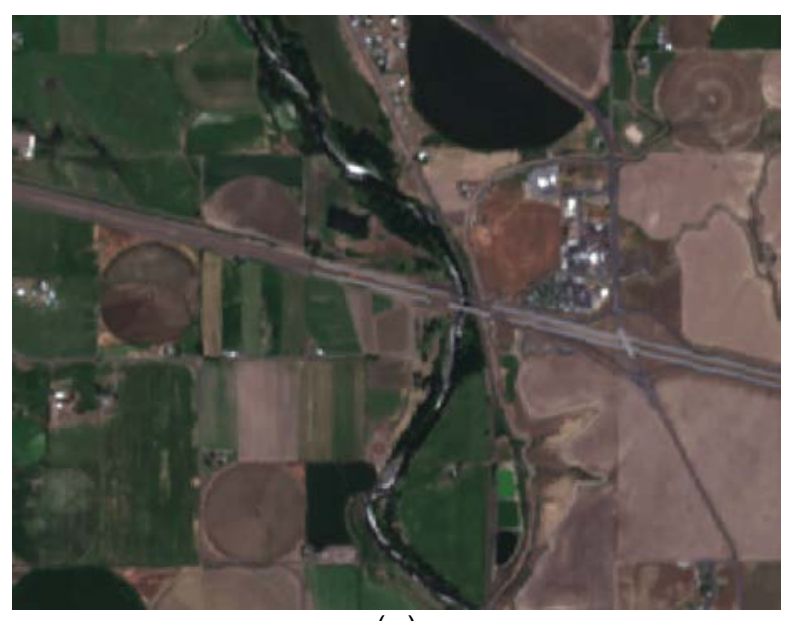

(a)

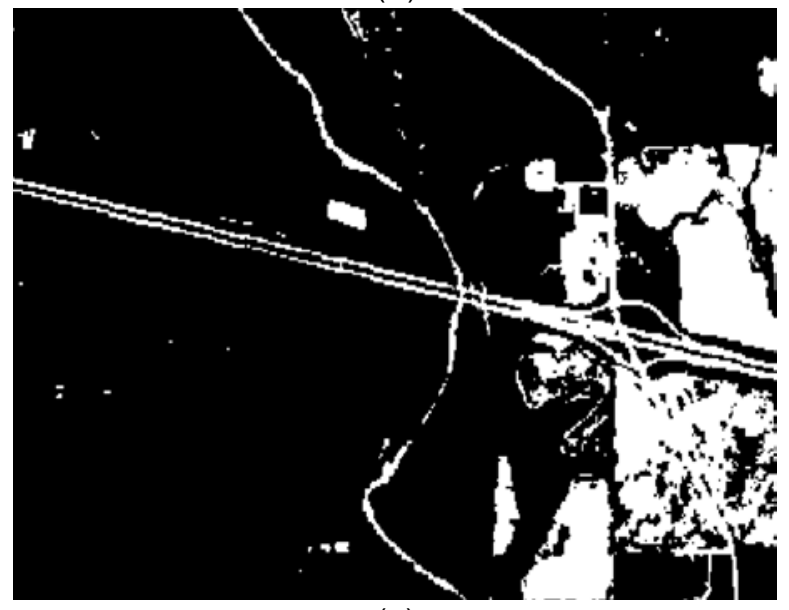

(c)

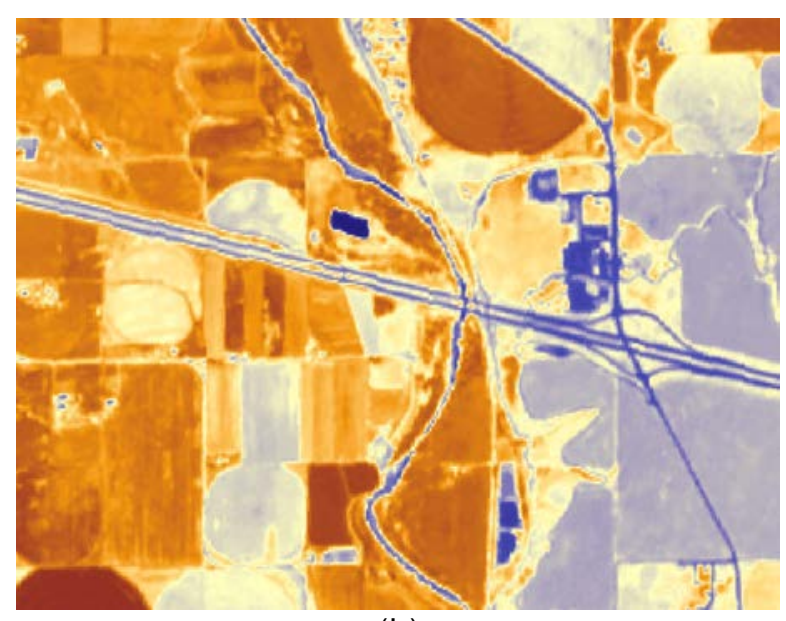

(b)

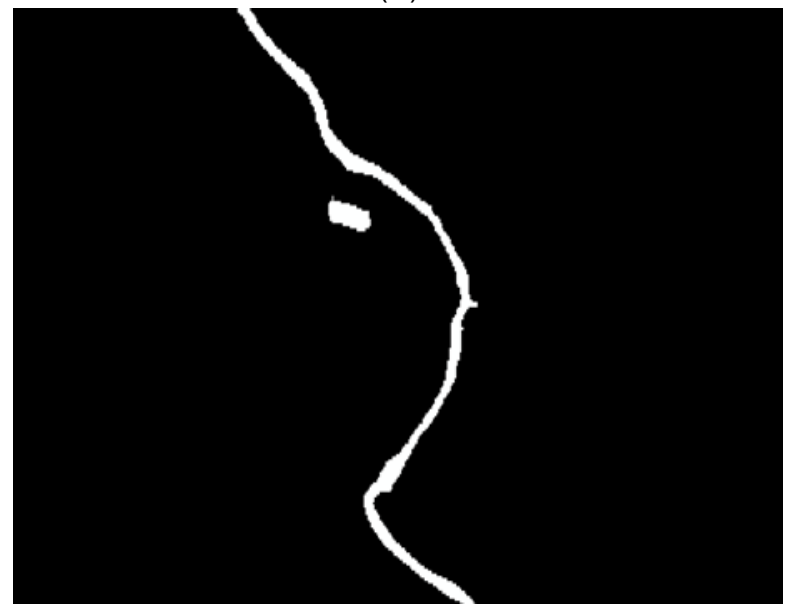

(d)

FIGURE 6.-Comparison of (a) Sentinel-2 RGB, (b) Colored NDWI, (c) Binarized NDWI using Threshold of -0.2 , and (d) Final Model Output. ${ }^{2}$

\section{Testing Data Metrics}

As a model performance evaluation, metrics generated on model testing data can be used for direct pixel-to-pixel comparison between model predictions and corresponding ground truth values. Each pixel thus has two axes: predicted, provided by the model, and actual, provided by manual ground truth data. In these binary classification typologies, pixels can have one of four labels: true positive (TP), true negative (TN), false positive (FP), and false negative (FN). Table 1 provides these testing metrics in a contingency table format.

\footnotetext{
2 Even though the NDWI threshold thumbnail lights up for many roadways and other nearby terrain, some river
} pixels are still below threshold. 
TABLE 1.-Confusion Matrix of Prediction Model on Ground Truth Data excluded from Training Pipeline for Performance Metrics (Percent are of All Pixels over the Study Region).

\begin{tabular}{|c|c|c|c|}
\cline { 3 - 4 } \multicolumn{2}{c|}{} & \multicolumn{2}{c|}{ Model } \\
\cline { 3 - 4 } Actual & \multirow{4}{*}{ Wet } & $\begin{array}{c}8,143,067 \\
(9.30 \%)\end{array}$ & $\begin{array}{c}102,573 \\
(0.12 \%)\end{array}$ \\
\cline { 3 - 4 } & \multirow{4}{*}{ Dry } & $\begin{array}{c}99,257 \\
(0.11 \%)\end{array}$ & $\begin{array}{c}79,231,679 \\
(90.47 \%)\end{array}$ \\
\hline
\end{tabular}

Statistical metrics for binary classification can build on these four categories to compute four statistical measures of model performance: accuracy, precision, specificity, and sensitivity (Table 2). Accuracy, the most universal metric, measures the percentage of all predictions that were correct. Precision measures the proportion of correct wet-pixel predictions out of all wet pixel predictions. Sensitivity measures the proportion of wet pixels that were predicted to be wet. Specificity measures the proportion of dry pixels that were predicted to be dry.

TABLE 2.-Binary Classification Statistical Metrics for Model Performance.

\begin{tabular}{|l|l|l|l|}
\cline { 2 - 3 } Accuracy & $99.77 \%$ & Specificity & $99.87 \%$ \\
\hline Sensitivity & $98.76 \%$ & Precision & $98.80 \%$ \\
\hline
\end{tabular}

\section{Active Learning Comparisons}

A distinct component of this study's machine-learning process was the active training approach. This iterative procedure allowed the team to use intermediary models to predict a single target extent, and then to compare the intermediary prediction to one produced by the final model. Figure 7 shows the progression of model outputs based on this description. Figure $7 \mathrm{~b}$ demonstrates an intermediary model misclassifying a railway yard as water, while the final model output corrects this result.

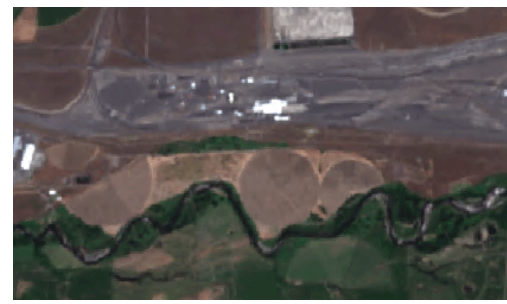

(a)

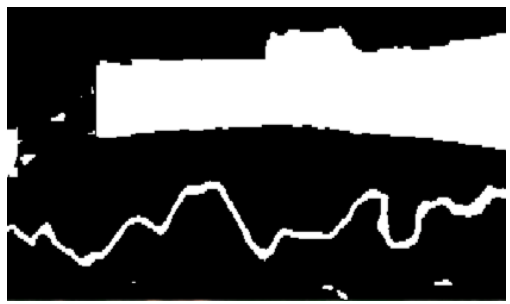

(b)

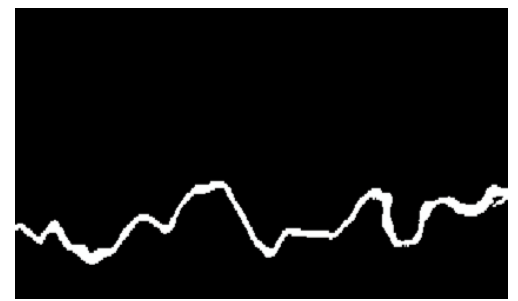

(c)

FIGURE 7.-Overview of Training Process. ${ }^{3}$

\section{Discussion}

Convolutional neural networks and linear classifiers are deep learning architectures that can learn directly from rasterized satellite data. Applying a hybridized model for the initial step of channel-width characterization demonstrates the value in employing deep learning techniques for handling complex, non-linear relationships in remote sensing datasets. In this study, positive trends in

\footnotetext{
3 Progression of model output for extent (a). Thumbnail (b) depicts an intermediary output. The region was used to in re-training to develop a final model. Thumbnail (c) depicts the final output for the region in June.
} 
machine learning methodologies for different types of prediction output led wet pixel detection in Oregon to employ a three-neural network approach, where each neural network tackled a separate domain problem.

Results show that the model developed in this study has a 99 percent accuracy on testing data. Though remarkable, some considerations are relevant in the assessment and discussion of the final dataset. A machine learning model is only as accurate as its training dataset. The river detection is limited by the resolution of public Sentinel-2 data, which has a 10-meter pixel resolution. River reaches with a 10 meter bank width will be encoded in the satellite data in an unpredictable manner, contingent on the alignment of the satellite grid over the physical reference plane. River reaches less than 10 meters may only be partially encoded in the satellite data (i.e., an average of adjacent physical features) or completely excluded.

In addition, a machine learning model can only predict river water in areas with characteristics that are as similar and diverse as those of its training dataset. Situated on the United States' Pacific Northwest coast, Oregon is made of topographically diverse provinces. Effort was invested during the collection of training data to represent a wide range of features across Oregon's various provinces, with ground truths encompassing river widths of 10 meters to more than 700 meters. However, a larger effort in developing diverse training data was constrained by the resources to complete the entire river geometry characterization process. Future models for this purpose will benefit from a thorough investigation of Oregon's river reaches and the variable surroundings that influence photometric measurements within the state.

\section{Centerline Extraction}

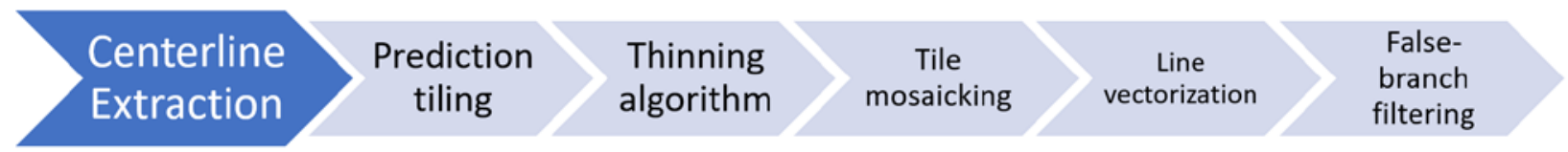

FIGURE 8.-Flow Diagram for River Centerline Extraction.

With a full-state raster map representing water presence predictions across the state, the next step in characterizing river widths is to accurately delineate centerlines within the positive prediction output. These centerlines are used for subsequent steps to position orthogonal line segments, which are crucial in the construction of a final river width dataset.

The methodology for extracting centerlines from the binary prediction map in this study uses the Scikit-image Python library and a systematic filtering approach. Scikit's morphological thinning method is relatively resource-intense and very sensitive to noise near the boundaries of encoded shapes, creating many "false branches." To solve these problems, the research team developed a technique where the prediction map is first tiled, thinned, and then mosaicked for featurization, which converts the raster data to vector data for the specific areas of interest (i.e., river reach segments). Once centerlines are in vector format, the team can then perform network analysis with a refined false-branch filtering algorithm to remove irrelevant segments. Figure 8 specifies the steps and shows the exact procedure, along with Python and library versions. Steps in this process include:

1. Full-state prediction mask is tiled into $4096 \times 4096$ images with 50 percent horizontal and vertical overlap

2. Tiles are processed using Scikit-image's "thin" algorithm in the morphology module in Python 3.7.5. 
3. Tiles are mosaicked, excluding 1024 pixels along each border

4. Mosaic is vectorized using ArcMap's "Raster to Polyline" toolbox

5. Centerline polyline processed by Python NetworkX library into nodes and edges

6. Edges are excluded based on satisfying one of two criteria based on edge's respective end nodes:

7. The edge has a 1-degree node and a 4-degree node

8. The edge is less than 1000 meters, has a 1-degree node, and a 3-degree node

9. Freehand removal of remaining erroneous centerline segments is performed across the $\mathrm{AOI}$

\section{Channel Width Characterization}

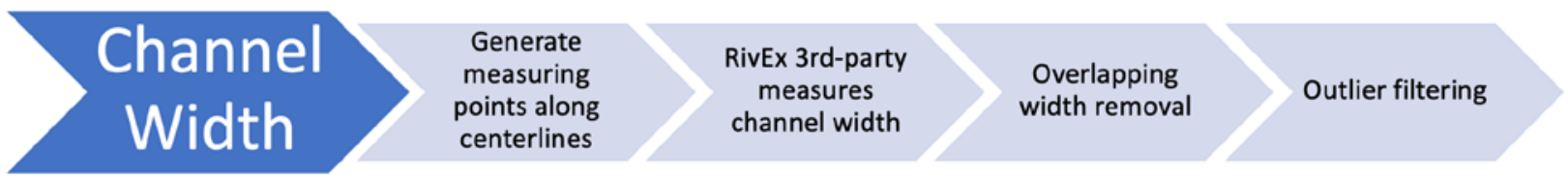

FIGURE 9.-Flow Diagram for Channel Width Derivation.

River centerlines along with a positive water prediction map provide the pillars for estimating the widths of river reaches across Oregon State. The research team used RivEx, a third party software, to create a vector dataset of river widths. Post-processing of river width lines is required to remove outlier widths, which often occur in cases where centerline perpendicularity fails to provide an adequate means of channel width estimates, as well as in cases near centerline branching, where width lines measure the reach of the intersecting river rather than the actual river's width. Figure 9 shows the procedure to estimate river width and identify and remove errors. This section will discuss briefly the preparation of inputs for RivEx, and then post-processing techniques used to improve RivEx's outputs.

\section{Data Preparation and RivEx}

RivEx requires three inputs: a centerline network, a corresponding channel polygon in which those centerlines fall, and a collection of points at which to conduct the width analysis. ArcMap's "Raster to Polygon" toolbox was used to convert the full-state channel mask into a vector representation. QGIS's "Points along geometry" algorithm was then used to generate points along each centerline feature at 30 meter intervals. RivEx draws a line segment at each point perpendicular to the centerline between edges of the river water body. The length of the line segment can then be calculated as the river width at that point.

\section{Outer Width}

Drawing line segments orthogonal to the centerlines could be complicated at locations near the confluences of rivers resulting in some line segments that are much longer than actual river widths. In order to eliminate these abnormal line segments, the team developed, tested, and implemented a method to identify width outliers. Width measuring points were pooled in groups of 20 along the centerline of each river reach from one end to the other. Groups that had fewer than 20 samples are 
merged with the preceding sample group. Width estimates greater than the $75^{\text {th }}$ percentile of the group were flagged as outliers.

\section{Near-branch Width Filtering}

Width estimation errors often arise from centerline branches. The team identified and tested a filtering method to minimize the errors by implementing the following steps for all three degree nodes in the centerline network.

1. Calculate the average width measurement associated with 10 nearest measuring points along every branch intersecting current node

2. Calculate the average of the two smallest averages from step \#1

3. Filter off measuring points on the edge with the largest average from step \#1 using 50 percent of the average width calculated in step \#2

\section{Channel Depth Projections}

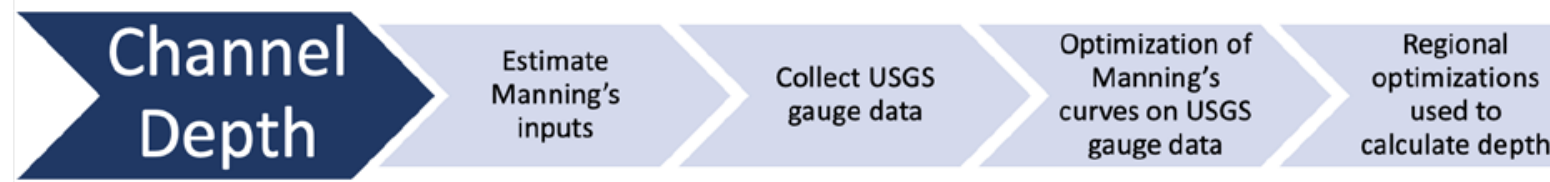

FIGURE 10.-Flow Diagram for Channel Depth Optimization.

The channel depths are determined using a hydraulic method, Manning's equation, with measurements available at each of USGS gauges collected from the USGS Groundwater and Streamflow Information Program. A trapezoidal model of river cross sections is assumed to optimize hydraulic parameters as well as river depths at the gauge. The model parameters at gauge (e.g., a bank angle or an angle that is critical to define the shape of trapezoidal cross-section) is generalized and extrapolated to any river reaches within the same or similar watershed characteristics. Figure 10 illustrates a process to derive river depth.

Input for Hydraulic Approach (Manning Equation)

$$
\begin{aligned}
& Q=\left(\frac{1.49}{n}\right) A R^{\frac{2}{3}} \sqrt{S} \\
& \text { where } R=\frac{A}{P_{\text {wetted }}}
\end{aligned}
$$

Manning's relationship (Eq. 2) can be represented using flowrate (Q), cross-sectional area (A), hydraulic radius (R), slope (S), and Manning's roughness coefficient (n). Slope and Manning's roughness are established at the beginning of the process and treated as known quantities throughout. Slope is taken from associated NHD Plus Version 2 flowlines. For measuring points throughout the state, points are associated with flowlines by performing a spatial join on nearest feature. For gauge stations, NHD Plus Version 2 flowlines are associated using joined attributes from USGS gauge database. 


$$
\begin{aligned}
& m=\left\{\begin{array}{cc}
1.00, & 1.0 \leq s<1.5 \\
1.15, & 1.2 \leq s<1.5 \\
1.30, & s \geq 1.5
\end{array}\right. \\
& n=n_{\text {base }} \times m
\end{aligned}
$$

Manning's roughness is estimated by multiplying a base roughness value associated with soil composition by a meandering coefficient $(m$, Eq. 3 ) where sinuosity $(s$, Eq. 4$)$ is calculated for each centerline segment (CR).

Soil composition is evaluated using data accessed through the NRCS STATSGSO dataset. Map units are composed of individual components, each with their own horizon layer information. For each component, the research team calculated soil grain size group percentages based on the second horizon layer's soil composition measurements (table 3). If no second horizon was listed, the first value was used. Map unit soil grain size aggregate percentages were then estimated by taking the weighted sum of each components groupings. Water, river wash, and badland component types were excluded from the weight values. The sum of each grouping was then used to derive a base Manning's roughness value $\left(n_{\text {base }}\right)$. The mapped roughness values from the largest-grain size category with weighted percentage plus any grain size category exceeding 50 percent were used as

\begin{tabular}{|c|c|c|c|}
\hline Group & Sample grain size & Horizon attributes used & $n_{\text {base }}{ }^{*}$ \\
\hline 1 & Less than $1 \mathrm{~mm}$ & $\begin{array}{ll}\text { - } & \text { sandvc_r } \\
\text { - } & \text { sieveno10_r } \\
\text { - } & \text { frag3to10_r } \\
\text { - } & \text { fraggt10_r }\end{array}$ & 0.025 \\
\hline 2 & $1 \mathrm{~mm}$ to $2 \mathrm{~mm}$ & $\begin{array}{ll}\text { - } & \text { sandvc_r } \\
\text { - } & \text { sieveno10_ } \\
\text { - } & \text { frag3to10_r } \\
\text { - } & \text { fraggt10_r }\end{array}$ & 0.0305 \\
\hline 3 & $2 \mathrm{~mm}$ to 4 & $\begin{array}{ll}\text { - } & \text { sieveno10_ } \\
\text { - } & \text { frag3to10_r } \\
\text { - } & \text { fraggt10_r }\end{array}$ & 0.032 \\
\hline 4 & 4 to 64 & - frag3to10_r & 0.040 \\
\hline 5 & Greater than 64 & - fraggt10_r & 0.055 \\
\hline
\end{tabular}
the entire map unit's roughness value $\left(n_{\text {base }}\right)$.

TABLE 3.-Interval Groups Calculated based on Grain Size Representative Percentage.

Optimizing Manning's Curve at Gauging Station

To approximate bank angles for the trapezoidal model at gauge, the Manning's curve at each station was used to fit the rating curve data provided by the USGS Ratings Depot. The rating curve at each 
station provides discharge rates at given gauge heights. The Manning's equation (area and wetted perimeter) was rewritten as functions of channel depth and width at an event $x$ (Eq 5). Using consistent properties for a trapezoidal model, the research team derived relationships for river crosssection and wetted perimeter during event $x$, given known geometries at a reference event $x=0$.

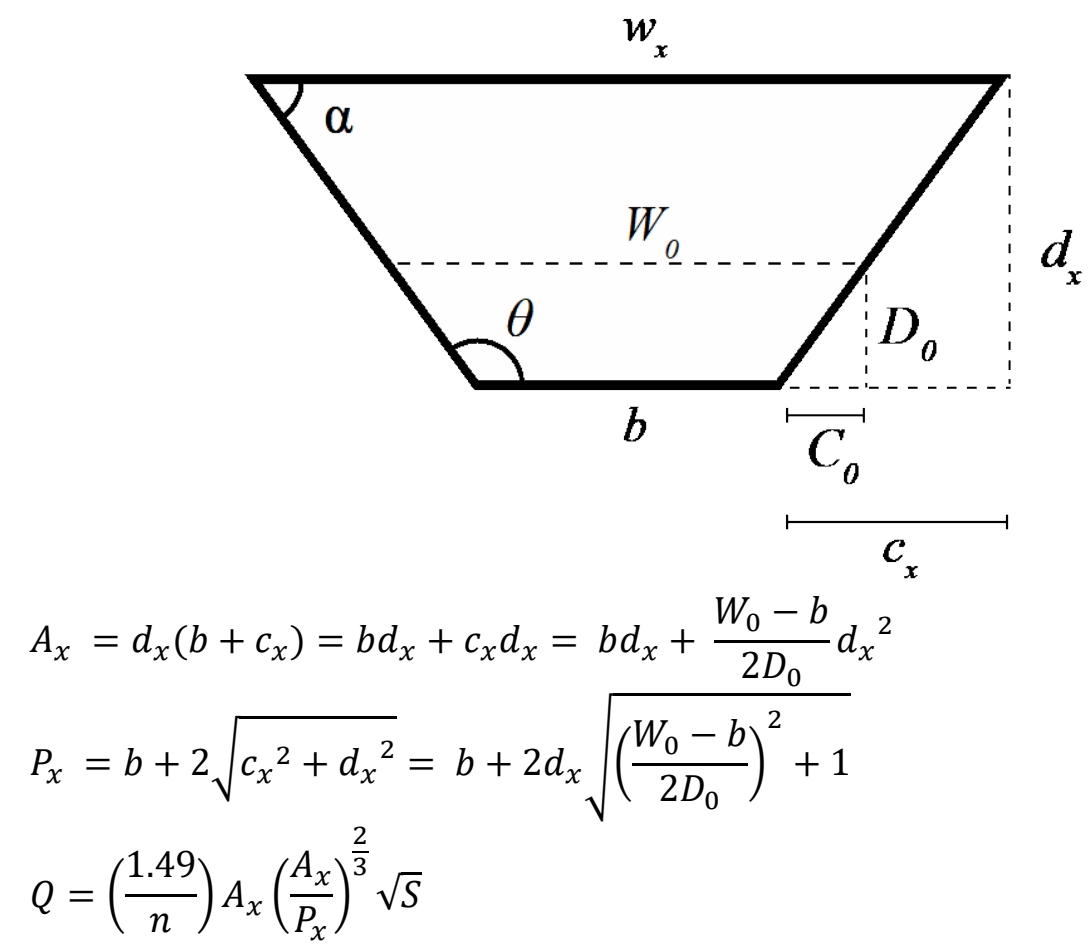

Eq. 5

Event $x=0$ is defined as the mean monthly river geometry in September. Width at $x=0$ is calculated by querying station field measurements after 31 December 2000 where corresponding recorded discharge rates fell within 10 percent of the September 2019 mean discharge (2018 and then 2017 data are used if more recent data is unavailable). The mean channel width $(\bar{W})$ of the resulting dataset at each station provided an approximation of channel width at $x=0$.

$$
A=W D_{\min } \quad A=\frac{1}{2} W D_{\max }
$$
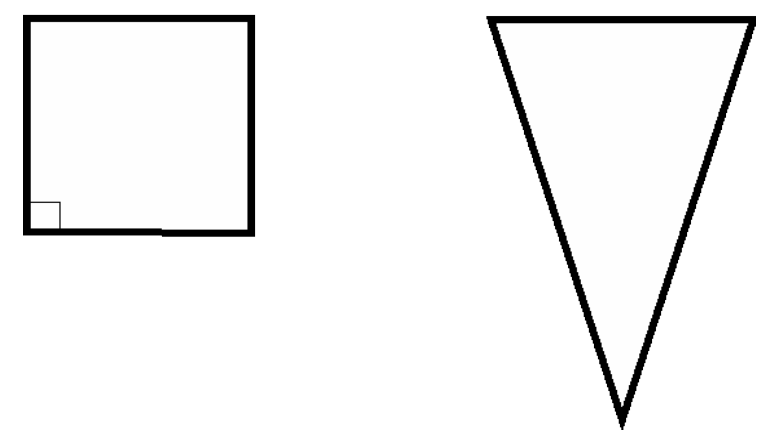

$$
D_{\min }=\frac{\bar{A}}{\bar{W}}
$$

Eq. 6 


$$
d_{x}=g_{x}+z
$$

Eq. 7

For channel depth at $x=0$, the research team used the USGS ratings curve to predict mean monthly gauge height from mean monthly discharge. Since gauge height can be a poor predictor for depth, particularly in streams where seasonal variation allows the reference height to be placed at only a fraction of the actual depth, a practical range for river depth was determined by considering the extremes of possible river geometries. The mean channel width $(\bar{W})$ and mean channel area $(\bar{A})$ from width derivation were used in Equation 6 to identify stations where the projected $D_{\text {min }}$ exceeded the historic gauge height. At these stations, the researchers set $z=D_{\min }-G_{0}$ (a variant of Equation 7), where $z$ represents the offset between the gauge's reference height and river's actual depth. At other stations, gauge height was accepted as a realistic approximation of river depth, (i.e., $z \approx 0$ ).

A non-linear least squares optimization was performed with the model function from Eq. 5 to fit discharge data directly from the USGS ratings curves and adjusted gauge height data. Parameters optimized through the regression analysis included base width $(b)$, slope $(S)$, and Manning's roughness $(n)$. Slope and Manning's roughness, though defined as constants at the beginning of the process, were given optimization ranges to allow for small amounts of error in the calculation process. Channel bank angle $(\theta)$ was derived with Equation 8.

$$
\theta=90^{\circ}+\tan ^{-1}\left(\frac{W_{0}-b}{2 D_{0}}\right)
$$

For stations where the researchers assumed of a nonzero $z$, an extra step for offset $z$ calibration was performed. The mean channel area $(\bar{A})$ representative of event $x=0$ was used to recalculate a $D_{0}{ }^{\prime}$ from which an updated offset, $z^{\prime}$, was derived ( $z$ in Equation 7$)$. The non-linear least squares optimization was run again, adjusting heights provided by the USGS gauge data using offset $z^{\prime}$. This calibration was repeated until the updated derived angle $\theta$ fell within 1 percent of the previous iteration's derived angle.

$$
\begin{aligned}
& b=W_{0}-\frac{2 D_{0}}{\tan \propto} \text { from } \frac{D_{0}}{\frac{1}{2}\left(W_{0}-b\right)}=\tan \propto=\tan \left(180^{\circ}-\theta\right) \\
& D_{0}=G_{0}+z \\
& A=\frac{W_{0}+b}{2} \times D_{0}=\frac{W_{0}+b}{2} \times\left(G_{0}+z\right)=\left(W_{0}-\frac{2\left(G_{0}+z\right)}{\tan \propto}\right) \times\left(G_{0}+z\right)
\end{aligned}
$$

\section{Predicting Measuring Point Depths}


conjunction with discharge rate groupings, to predict channel bank angles at each measuring point (see table 4 for breakdown of methodology).

TABLE 4.-Method for Obtaining Measuring Point Channel Bank Angle Based on Measuring Point Discharge at $x=0$.

\begin{tabular}{|c|l|l|l|}
\hline Group & \multicolumn{1}{|c|}{ Sample grain size } & \multicolumn{1}{|c|}{ Horizon attributes used* } & \multicolumn{1}{c|}{ Spatial Unit } \\
\hline 1 & Less than 100 & $\begin{array}{l}\text { Average of station angles where } \\
Q_{0, s t a t i o n}<100 c f s\end{array}$ & $\begin{array}{l}\text { Nearest HUC-8 (in } \\
\text { same HUC-4*). }\end{array}$ \\
\hline 2 & 100 to 500 & $\begin{array}{l}\text { Average of station angles where } \\
100 c f s \leq Q_{0, \text { station }}<5000 c f s\end{array}$ & $\begin{array}{l}\text { Nearest HUC-8 (in } \\
\text { same HUC-4*). }\end{array}$ \\
\hline & 500 to 2000 & $\begin{array}{l}\text { Average of station angles where } \\
500 c f s \leq Q_{0, \text { station }}<2000 c f s\end{array}$ & $\begin{array}{l}\text { Nearest HUC-8 (in } \\
\text { same HUC-4*). }\end{array}$ \\
& & $\begin{array}{l}\text { In Eastern part of state, the } \\
\text { average of the entire region is used } \\
\text { instead }\end{array}$ & \\
\hline 4 & Greater than 2000 & $\begin{array}{l}\text { Average station where } Q_{0, \text { station }} \geq \\
2000 c f s\end{array}$ & AOI \\
\hline
\end{tabular}

For deriving channel depth, the research team used the model in Eq. 5 with updated area and parameter functions (Eqns. 11 and 12) to solve for depth.

$$
\begin{aligned}
& A_{x}=d_{x}\left(w_{x}-\frac{d_{x}}{\tan \alpha}\right) \\
& P_{x}=b+2\left(\frac{d_{x}}{\sin \alpha}\right)=w_{x}-\frac{2 d_{x}}{\tan \propto}+\frac{2 d_{x}}{\sin \alpha}
\end{aligned}
$$

Eq. 2

\section{Data Summary and Limitations}

This project generated two sets of results-one for September and one for June-using the RCT tool developed in this study. These two time periods were selected to evaluate effects of annual low stream flows (September) and high stream flows (June) on river water width and depth characteristics. The final data sets include variables of river widths, depths, and flow rate for each time period for all rivers across Oregon, and organized in GIS format for further analysis of the effects of river barriers on emergency response and recovery efforts following a CSZ earthquake as part of the Oregon Transportation Systems RRAP project.

The accuracy of channel widths and depths predicted by this tool depend greatly on the resolution of satellite data used in the input training pipeline, as well as the uncertainty associated with linearly optimized depth data. In this project, the resolution of 10 meter satellite spectral data was used. With more high-resolution satellite data (e.g., 1 meter) publicly available in the near future, this tool could provide significantly increased accuracy of river widths and could take advantage of automated processes leveraging the computational power of new high performance graphics processing unit (GPU)-based supercomputing systems. 


\section{Acronyms and Abbreviations}

$\begin{array}{ll}\begin{array}{l}\text { AOI } \\ \text { Argonne }\end{array} & \begin{array}{l}\text { Area of Interest } \\ \text { Argonne National Laboratory } \\ \text { CISA }\end{array} \\ \text { CSZ } & \begin{array}{l}\text { Cybersecurity and Infrastructure Security Agency } \\ \text { Cascadia Subduction Zone }\end{array} \\ \text { DNN } & \text { Deep Neural Networks } \\ \text { HUC } & \text { Hydrologic Unit Code } \\ \text { mNDWI } & \text { Modified Normalized Difference Water Index } \\ \text { NDWI } & \text { Normalized Difference Water Index } \\ \text { NHD } & \text { National Hydrography Dataset } \\ \text { NIR } & \text { Near Infrared } \\ \text { RCT } & \text { River Characterization Tool } \\ \text { RRAP } & \text { Regional Resiliency Assessment Program } \\ \text { SWIR } & \text { Shortwave Infrared } \\ \text { USGS } & \text { U.S. Geological Survey } \\ \text { WPDM } & \text { Wet Pixel Detection Model }\end{array}$




\section{References}

Acharya, Tri Dev, Anoj Subedi, Dong Ha Lee, 2018, "Evaluation of Water Indices for Surface Water Extraction in a Landsat 8 Scene of Nepal," Sensors (Basel). 18(8):2580. Published August 7. doi:10.3390/s18082580

Bieger, K., Rathjens, H., Allen, P.M., Arnold, J.G., 2015. "Development and Evaluation of Bankfull Hydraulic Geometry Relationships for the Physiographic Regions of the United States." Journal of the American Water Resources Association. 51(3): 842-858

Castro, J. (1997). "Stream classification in the Pacific Northwest: methodologies, regional analyses, and applications." Oregon State University.

CISA, 2021, Resiliency Assessment: Oregon Transportation Systems.

Fenneman, N.M., and Johnson, D.W., 1946, "Physiographic divisions of the coterminous U.S," U.S. Geological Survey. Scale 1:7,000,000.

Fenneman, N. M., 1928, "Physiographic divisions of the United States." Annals of the Association of American Geographers 18(4): 338-342.

Huang, Chang, Yun Chen, Shiqiang Zhang, and Jianping Wu, 2018, "Detecting, extracting, and monitoring surface water from space using optical sensors: A review," Reviews of Geophysics 56: 333-360.

McFeeters, S.K., 1996, "The use of the Normalized Difference Water Index (NDWI) in the delineation of open water features," International Journal of Remote Sensing 17(7): 1425-1432

National Park Service, undated, "Physiographic Provinces," https://www.nps.gov/articles/series.htm?id=12C422BC-1DD8-B71B-0B1552CA902E5909, accessed July 15, 2021.

Radford, Alec, Luke Metz, Soumith Chintala, 2016, “Unsupervised Representation Learning with Deep Convolutional Generative Adversarial Networks," ICLR 2016.

Xu, Hanqiu, 2006, "Modification of normalized difference water index (NDWI) to enhance open water features in remotely sensed imagery," International Journal of Remote Sensing 27(14): 3025-3033. 


\section{Argonne}

\section{Decision and Infrastructure Sciences Division}

Argonne National Laboratory

9700 South Cass Avenue, Bldg. 203

Argonne, IL 60439

www.anl.gov

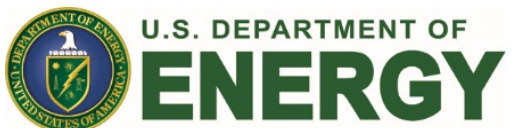

Argonne National Laboratory is a U.S. Department of Energy laboratory managed by UChicago Argonne, LLC 